\title{
Más allá de la investigación cualitativa...
}

\author{
Lewis Pereira ${ }^{1}$
}

\section{Introducción}

Más allá de la investigación cualitativa tradicional se encuentra la superación de las limitaciones del positivismo, al menos dentro de las ciencias sociales, y la integración metodológica o la propuesta de que las metodologías se combinen en un conjunto coherente donde los investigadores utilicen las técnicas que consideren adecuadas (muchas de ellas creadas in situ), con el sólo propósito de conseguir las "pruebas" o la información empírica necesaria, sin preocuparse por las disputas epistemológicas del tipo de las aparecidas en la segunda mitad del siglo XX; es decir, inmersos en una concepción avanzada de la ciencia que deje de poner límites o mejor, que permita una mirada centrada en las demostraciones "fuertes" sobre la que los científicos sociales puedan estar de acuerdo. Y pudiera ser que dicha superación no haya que promoverla demasiado, sino que ya se encuentra en marcha, de manera que a quienes habrá que dirigir el reclamo sea a los epistemólogos, sociólogos y antropólogos de la ciencia que la siguen considerando un problema. Hace algunos años ya, los trabajos que combinaban métodos representaban el 23,5\% en Norteamérica y el 21,5\% en Venezuela (Ruiz Bolívar, 2008, p. 18). El objeto, por tanto, de este breve capítulo no será el quehacer de los científicos sociales, sino el enfoque de los que escriben sobre ella.

Muchos piensan que lo que se encuentra más allá es la investigación mixta, pero otros, que se trata de los multimétodos o la investigación multimodal, una investigación que combina los métodos sin los paradigmas y sin tener en cuenta su discusión

1 Doctor en Antropología. Maestría en Antropología Social. Sociólogo. Docente invitado en la Universidad de Cartagena (Colombia). Miembro del Grupo de Investigaciónn "Ciencia, Tecnología y Sociedad" avalado por COLCIENCIAS. Correo: pereira.lewis@gmail.com. 
como algo importante (Ruiz Bolívar, 2008). Sin embargo, ¿Es cierto que al salir de la investigación cualitativa hay que abandonar sus aportes para la ciencia social? y si no es así ¿De qué se estaría hablando exactamente? ¿A qué tipo de premisas epistemológicas se estaría haciendo referencia?; ¿Una investigación "multimétodo" es una investigación mixta enriquecida? ¿Se podría considerar que se trata de una investigación cualitativa mejorada...?, y ¿Qué sería una investigación cualitativa mejorada? o ¿En qué consiste? Una discusión seria tiene que comenzar por el problema de la integración. Veamos las ideas esenciales o los esbozos del paradigma emergente que ahora se percibe en el horizonte.

\section{La adscripción necesaria}

Aunque no sea reconocido del todo por los practicantes de oficio, la investigación mixta tiene que ver con algo que no resulta cómodo, a saber, la combinación de los supuestos epistemológicos de la ciencia "comprensiva" en el sentido Schutziano con los de la ciencia positivista de la primera mitad del siglo XX (el positivismo lógico); y la impresión que se tiene en América Latina es que para los profesores de metodología y los tutores de tesis de grado de las universidades, estos supuestos son irreconciliables, lo cual se ve reforzado por abundante literatura sobre el tema (Ver las discusiones sobre el tema en Ruiz Bolívar, 2008; Cook \& Reichardt, 1986).

Para unos $-y$ es uno de los principios básicos de la ciencia cualitativa-, el estudio científico de la realidad humana requiere la "comprensión" de las estructuras de significado en las que se sumerge el comportamiento y subjetividad de los seres humanos, o la concepción de que la vida humana implica la participación en una "red de significados", como una tela de araña, en la que se suspenden las acciones y los pensamientos de una manera tal que sin la misma aquellos pierden sentido. La realidad construida por las personas, a diferencia de la realidad inanimada estudiada por los científicos naturales, es teleológica y guiada por cánones morales. El amor, por ejemplo, forma parte de esa realidad, aunque no sea un elemento propio de los átomos y las rocas; de allí, pues, la problemática de la declaración realizada por el primer Wittgenstein en plena efervescencia 
del positivismo lógico, cuando llegó a expresar en el aforismo 6.41: "El sentido del mundo tiene que quedar fuera de él. En el mundo, todo es como es y todo sucede como sucede..." (Wittgenstein, 2007, p. 269)

Se podría afirmar, de hecho, que allí se encuentra el principio de la "ciencia de los objetos calculables" dedicada a una objetividad exterior, suponiendo una trama de relaciones entre objetos inanimados, sin teleología, ni símbolos y vivencias porque las rocas y los átomos no tienen ese tipo de atributos (Padrón, 2007). Claro que, en este caso, mal podría el investigador proyectar su propia teleología, símbolos y subjetividad, de acuerdo a este tipo de ciencia tales aspectos no pueden formar parte del conocimiento científico y que no tiene sentido su investigación. Se debe recordar a Barrhus Skinner, el padre el conductismo operante, y sus conocidas tesis sobre el estudio del comportamiento como algo externo, matematizable y remitido a variables observables, y su disputa con Jean Piaget. En contraposición la "ciencia de los objetos vivibles", como la denomina el profesor José Padrón, o la ciencia para la cual se hace necesaria la captación de las vivencias, los sistemas de significados y representaciones del mundo, se encargaría del mundo humano.

Se trata de una diatriba básica, es decir, la investigación teniendo por delante los significados y la teleología (con intención y voluntad) y una que no los tome en cuenta. Para muchos una postura centrada en los objetos calculables impide el acercamiento a una sobre los objetos vivibles; sin embargo, la relación entre técnicas de investigación y paradigmas, y entre los datos y los paradigmas agrega un elemento adicional a la problemática: ¿están las técnicas y los datos necesariamente adscritos a los paradigmas? ¿Hay una adscripción necesaria entre ellos por lo cual la investigación mixta si es una combinación de paradigmas? Esta discusión podría ser importante porque si la adscripción no es necesaria entonces, comienza a disolverse la oposición entre paradigmas, esto es, entre los datos cuantitativos y las ideas sobre la objetividad externa, ausencia de sentido en el mundo e información cualitativa a partir de criterios sobre el comportamiento humano contextualizado. 
De entrada, da la impresión de que la respuesta es positiva, que los métodos si están necesariamente vinculados a los paradigmas, pero es muy probable que se puedan argumentar juicios eficaces contra tales suposiciones que permitan problematizarla suficientemente.

\section{Una experiencia personal}

Cuando yo era estudiante de pregrado en una universidad venezolana tuve la fortuna de llegar a formar parte de un centro de investigaciones históricas y trabé relaciones íntimas con el mundo de los historiadores. Se hacía historia regional a un nivel de detalle y precisión notables, todos los días había que salir a buscar datos en los centros de documentación, bibliotecas privadas y públicas de la ciudad, y aunque no era mi área natural de estudio, porque yo era estudiante de sociología, pronto me familiaricé con mis tareas, las que realizaba con verdadera pasión de joven investigador. Revisé incontables documentos de los siglos XIX y XX, algunos en muy mal estado, que traían todo tipo de datos, debía revisar listas de estudiantes inscritos en la universidad sobre la cual se hacía la investigación (se trataba de un proyecto de historia de la educación superior), programas de asignaturas, notas de prensa en los periódicos locales sobre las actividades sociales de los profesores universitarios, documentos oficiales sobre nombramientos políticos, publicidad relacionada con el entorno social, cuadros estadísticos sobre exportaciones de café, etc., y en todos los casos, había que procesar la información de diferentes formas. Recuerdo haber tenido unas fichas en cartulina, que se habían elaborado especialmente para el proyecto, con los ítems requeridos para vaciar la información, y el análisis o el procesamiento de la información se hacía sobre esas fichas, que a veces contenían datos cualitativos pero otras, datos cuantitativos. $\mathrm{Y}$ recuerdo, de la misma forma, un momento memorable en el que llevaba meses a la caza de una carta o cualquier documento sobre lo que había sucedido en un período o un lapso de tiempo específico (había dudas sobre lo que había ocurrido o mejor, nadie sabía nada, ni siquiera los historiadores de profesión), y por casualidad, luego de tanto buscar, di con una carta que permitió esclarecer todo. Llegué con el documento transcrito al centro de investigaciones, como 
quien llega con un tesoro en las manos, y me dispuse a mostrárselo a las profesoras con las que trabajaba, les expliqué el hallazgo y en las semanas siguientes se hizo notar la importancia de la misma. El contenido permitía saber lo que había ocurrido, y se sabía por lo que en Historia se conoce como "crítica de fuentes" o "crítica documental" que combina análisis de contenido simple con examen del contexto histórico. Es una de vías por las que el historiador normalmente llega a sus hallazgos, a saber, comparando el contenido de los documentos con aquello que indican otras fuentes y el contexto histórico estudiado. Esto me fue persuadiendo de un tipo de metodología.

En otros momentos, se sometía a análisis sistemático las Memoria y Cuenta de los ministros de Instrucción Pública, que era como se llamaban los ministros de educación, triangulando dicha información con otros documentos y en el camino se elabora información parcial. Se podían construir tablas y polígonos de frecuencia, o se hacía análisis de contenido del tipo señalado. Y recuerdo que las nociones de "mentalidad histórica" y "contexto cultural" estaban presentes casi siempre porque eran necesarias, no se recurría a la antropología, pero si a categorías que guardaban relación con esa ciencia. Y desde siempre noté el significado profundo de la lejanía del tiempo histórico. Un investigador cree que puede pensar el pasado como el presente, pero se equivoca; el pasado es un mundo aparte para el cual no estamos preparados y donde solo funcionan las categorías propias de ese mundo.

En otras ocasiones, si no se tenía certeza sobre el lugar donde se encontraba sepultado algún rector de la universidad, iba al cementerio a buscarlo y a tomar fotografías e, incluso, ensayé cosas más exóticas como una historia basada en las biografías. Un taller dado en ocasión del paso por Venezuela de un francés prominente, que luego escribió el libro más importante que existe sobre la Revolución Mexicana, Xavier Guerra, nos persuadió de la importancia de la prosopografía y el uso de los computadores, por lo cual resultó de interés en adelante el seguimiento de los apellidos y la actividad social y política de los individuos. Por lo demás, esta universidad fue cerrada prematuramente, precisamente, por la actividad política de sus profesores. Me refiero a la Universidad del Zulia, clausurada en 
1903, sobre cuya historia se escribieron varios tomos en la que tuve oportunidad de participar (Rincón, Gamero, \& Ortín, 1986).

Por aquellos días yo no prestaba la más mínima atención a las divisiones de paradigma olas disputas entrelainvestigación cualitativay cuantitativa, -en realidad, nadie en el centro de investigación lo hacía-, y yo me formé con esa impresión. Los datos no estaban vinculados a paradigmas, sino que eran usados para realizar demostraciones, conseguir pruebas, y nada más. No veía conflictos entre la sociología y la Historia e intercambiaba las epistemologías, aplicaba a la Historia las categorías de la sociología y trataba la sociología como un tipo de Historia, en un cierto sentido, claro está. En realidad, yo no me daba cuenta que ya esto había sido ensayado desde el año 1929, con una experiencia mundialmente conocida como la Escuela de los Annales y que los problemas de investigación histórica me habían puesto en el camino de la investigación antropológica. Cuando los datos y la información recolectada corresponden a una sociedad que no es la nuestra, que se encuentra alejada de nosotros, ese es el resultado.

Cada dato suministra la oportunidad de un conocimiento, y años después, cuando me dispuse a enrumbar mi vida hacia la antropología y realicé mi primer postgrado, repetí las mismas estrategias. La gente no lo sabía, pero yo ya estaba familiarizado con la tarea, terminé el grado de magíster con una tesis que combinaba datos estadísticos con información cualitativa del tipo "compresión de las estructuras de significado". De hecho, me tomé bastantes libertades y realicé experimentos callejeros para apreciar las reacciones de las personas frente a unos dibujos, quería descubrir el modelo de las representaciones sobre los edificios de los museos, y la tesis doctoral, también en antropología, no hizo sino repetir la historia (Pereira, 2000).

Lo que parece que sucede, en el caso del historiador, es que las limitaciones impuestas por el tipo de registro al que tiene acceso lleva la epistemología de las ciencias sociales comprensivas al extremo. El historiador debe comprender el tiempo histórico que estudia, pero debe hacerlo con parte, solo una parte, de los restos materiales que quedaron. Se podría pensar que a la arqueología le ocurre lo mismo, es una ciencia de lectura de textos culturales, pero hecha desde los 
restos materiales dejados por las sociedades, algo parecido a lo que le ocurre a un detective o científico forense cuando llega a la escena del crimen y debe intentar reconstruir los hechos cuando ya todo pasó y la actividad humana que originó aquella escena ya ha cesado. Se tiene, en este caso, el problema de que lo único con lo que se cuenta es con la "huella" de la actividad. El extremo se produce porque no es la forma habitual de acercamiento para una ciencia comprensiva, al menos no como la imaginan los antropólogos especialistas en símbolos y los investigadores cualitativos de la hermenéutica o la fenomenología. El científico forense no tiene la oportunidad de ver los hechos mientras ocurren, al igual que el arqueólogo y su forma más tradicional, el historiador.

Los representantes de todas estas ciencias, en consecuencia, no pueden dedicarse a la lectura de símbolos emitidos directamente por los seres humanos porque no cuentan con ese material. En estos casos, se deben evaluar detenidamente las circunstancias en la que se encuentren los objetos materiales (piezas arqueológicas y documentos) y esto significa un trabajo en sí mismo que puede conducir al científico a la geología, biología, botánica o la química, y en el camino se realizan pruebas de ADN, Carbono 14, estudio de suelos, indagaciones de paleofauna, paleobotánica, etc., todo para preparar la lectura cultura final. Siendo así, la estrategia lleva por caminos diferentes a los historiadores, los arqueólogos respecto a los etnógrafos, fenomenólogos, sociólogos comprensivos, etnometodólogos y otros científicos. El hecho de que tenga que incluir a las ciencias naturales en su labor lo conduce, en parte, a la ciencia de los objetos calculables, cuando su suelo se relaciona más con la ciencia de los objetos vivibles.

El historiador debe ser capaz de imaginar las "estructuras de significados" del pasado para realizar adecuados análisis de datos cuantitativos o cualitativos. La necesidad de la interpretación hecha en los términos del tiempo histórico, queda demostrada en ejemplos relacionados con las estadísticas que ocasionalmente se consiguen en el mundo antiguo. Cipolla (1981, p. 83), un estudioso del período previo a la Revolución Industrial, hace referencia a esto argumentando que si se quieren analizar datos estadísticos del pasado, pongamos 
por caso, relativos a las "Amas de Cría", un fenómeno cultural de finales de la Edad Media, se deben comprender primero tales estructuras de significado. Las mujeres que prestaban sus servicios como amamantadoras de niños recién nacidos de familias ricas, que existieron en diferentes países europeos, no eran ni un servicio doméstico en el sentido actual, ni a una tercerización de ese sector, al igual que la prostitución.

Lo que, en todo caso, quiere decir esto es que la ciencia histórica se ve obligada a un análisis "comprensivo" previo si quiere entender datos cuantitativos, pero además, que los registros empíricos de los cuales se hace lectura, implican un trabajo que muchas veces la vincula a las ciencias naturales, es decir, algo como afirmar que antes de hacer ciencia interpretativa se debe realizar ciencia natural o que los datos objetivos de la matemática requieren una lectura semiótica. Y esta labor ha sido realizada, y la realizan hoy en día, los historiadores sin los supuestos tradicionales del positivismo o de la ciencia comprensiva.

Esto tiene implicaciones para la epistemología de las ciencias sociales: sus representantes no pueden plantearse la meta de hacer sólo ciencia comprensiva tomada directamente de la actividad humana, a menos, claro está, que se entienda que los registros empíricos para hacer eso son los mismos en la Historia que en la sociología comprensiva weberiana o la antropología simbólica de Geertz, y esto sí es algo un poco más problemático.

Y aun así, queda por delante el mundo de las matemáticas. Todas las ciencias sociales acuden a ellas, desde la antropología hasta la psicología, pasando por la economía, por lo cual no es posible hacer prosperar una propuesta avanzada de ciencia cualitativa si no se comprende este asunto y se da respuesta al mismo. Los historiadores acuden a la estadística y se diría que un enfoque integrado y superador pasa por dar una respuesta adecuada a los defensores de las matemáticas. Es conveniente, pues, acercar un poco más la mirada. 


\section{Lo que hacen los científicos sociales cuando matematizan}

Se puede relacionar el consumo de café con la frecuencia de las relaciones sexuales en una población o el consumo de leche de vaca durante la lactancia con la prevalencia de miopía en la edad adulta, y el tamaño de tierras cultivadas con el auge o declive de una civilización, y en todos los casos, se pueden aprovechar las herramientas desarrolladas por la estadística en la primera mitad del siglo XX, en buena medida gracias a Fisher y Pearson. Por otro lado, algunas veces se trabaja con algoritmos que permiten establecer la evolución del lenguaje desde hace varios millones de años hasta nuestros días o la difusión de rasgos culturales entre diferentes sociedades, al estilo de cómo se hacen los estudios de memes a partir de las ideas iniciales de Richard Dawkins. En realidad, ya los cálculos relativos a la relación entre la cantidad de tierras cultivables y el auge o caída de las civilizaciones a través de la historia, o del tamaño de la población respecto a la aparición de cierto tipo de tecnologías, o de la cantidad de especies salvajes disponibles para la domesticación y el porcentaje de especies que efectivamente domesticadas, forman parte de los avances de la corriente materialista de la antropología y algunos libros divulgadores han alcanzado gran madurez (ver la obra de Diamond, 2006, pp. 66-67).

Si se mira con atención se trata de dos tipos de investigación, a saber, por un lado, la relativa a la estadística aplicada a las relaciones entre variables, y por otro, la formulación de algoritmos sobre evolución. Las relaciones entre variables no son relaciones entre personas, pero esto no disminuye su valor, como no se trata de relaciones sociales no puede dar cuenta de las estructuras de significado de fondo que acompañan la actividad humana, pero sí, en cambio, de las relaciones entre aspectos aislables en el orden social; pongamos por caso, los vínculos entre el consumo de algún alimento y los hábitos sociales, o entre la tasa de suicidio y el nivel de ingresos económicos familiar, etc. ¿A qué se refiere, entonces, esto?

Las matemáticas no tienen conectores que sirvan para describir las relaciones sociales y los intercambios simbólicos, mucho menos las vivencias que se tienen durante dichos intercambios, por lo cual no sirven para la ciencia comprensiva. Si digo, por ejemplo, que 
"2 $+3=5$ " describo el mundo a través de esas cualidades, y los conectores ("+" y "=") son muy limitados. Los números, por otro lado, representan signos que pueden ser sustituidos por personas, árboles o lápices. La idea de dos personas a las que se les han sumado otras tres para conformar cinco, cabe dentro de la expresión, tanto como cinco árboles dentro de un parque. Cuando digo " $2+3=5$ ", ciertamente, estoy diciendo algo, pero no estoy haciendo referencia a casos particulares.

Si utilizara otros conectores y otros elementos abstractos como cuando digo "Luis ama a Mary, por lo cual viven juntos", encuentro que no hay fórmula matemática posible para expresar eso, ni siquiera si me refiero a todos los hombres que aman a sus mujeres. En las matemáticas no existen conectores para describir una relación amorosa, ni siquiera si sustituyo los nombres propios por símbolos como A y B, para decir algo como, "A ama a B => viven juntos". El conector para la relación de amor no está previsto (al menos no hasta ahora) en la matemática o en la lógica.

En los días de Bertrand Russell, a comienzos del siglo XX, se intentó resolver el problema diciendo cosas como "A $\wedge$ B => C", pero dicha expresión resulta tan general que dentro de la misma podría caber la expresión "Luis odia a Mary a pesar de que viven juntos", tanto como "Luis ama a Mary, por lo tanto, viven juntos". Los lógicos no distinguen esto, pero los científicos sociales si comprenden la diferencia. En las ciencias políticas se entiende, por ejemplo, que el odio colectivo puede arrastrar a los pueblos hacia consecuencias lamentables, y que su contrario, el amor o la veneración a un líder, a otro tipo de consecuencias. Si el ser humano, cualquier ser humano, pudiese ser sustituido por letras, los conectores,,$+-=,<,>, \times, \div$ serían demasiado rudimentarios e ambiguos para describir relaciones humanas.

Lo que esto significa es que las matemáticas no pueden ser adecuadas para dar cuenta de los "objetos vivibles" de la ciencia comprensiva, aunque si para relacionar variables. Se puede estudiar a nivel mundial la relación entre el nivel de felicidad y la esperanza de vida, o entre la fecundidad y el nivel de ingresos socioeconómicos de la mujer, y eso deben ser aportes al conocimiento científico. La 
ciencia comprensiva se encargará de lo demás, de los significados que circulan entre las personas y de las vivencias y sentimientos con los que miran su existencia, y sobre todo, de la estructura de significados que rige la sociedad. Del significado profundo de esta estructura ha dado cuenta la obra "La construcción Social de la Realidad" de Peter Berger y Thomas Luckmann (1968).

Entender el espacio al cual pertenecen las matemáticas en las ciencias sociales es esencial para comprender sus aportes al conocimiento científico. No se trata de que no sirva para nada, en todos los casos se trata de conocimiento para el orden social. Así, pues, descripciones como la de Heródoto quedarán a salvo de la matematización: En una ocasión hablaba de la vida cotidiana de los egipcios del siglo $\mathrm{V}$ antes de Cristo, una de las primeras descripciones etnográficas que se conocen, en los siguientes términos:

Supersticiosos por exceso, mucho más que otros hombres cualesquiera, usan de toda especie de ceremonias, beben en vasos de bronce y los limpian y friegan cada día, costumbre a todos ellos común y de ninguno particular. Sus vestidos son de lino y siempre recién lavados, pues que la limpieza les merece un cuidado particular, siendo también ella la que les impulsa a circuncidarse, prefiriendo ser más bien aseados que gallardos y cabales (Heródoto, 2006, p. 197).

\section{La paradoja de la objetividad}

Otra pregunta muy importante tiene que ver con la objetividad, ya que ella guarda relación con el problema de fondo referido a los puntos centrales de la discusión con el positivismo: el no reconocimiento de los estados subjetivos y la relación misma sujeto-objeto. El enfoque cualitativo explota al máximo la idea de la investigación social como una relación sujeto-sujeto, pero lo hace hasta el punto de proclamar una epistemología propia que la separa del resto de la ciencia. Para conseguir una confirmación sólo debe pensarse en las tesis fundamentales de Dilthey sobre la separación entre las ciencias de la Naturaleza y las del espíritu, y las ideas de libros de texto, como el de Steven Taylor, Robert Bogdan y Jorge Piatigorsky 
(1987), que asignan a este campo características no tradicionalmente vinculadas a la ciencia:

Los métodos cualitativos son humanistas. Aprendemos sobre los conceptos tales como belleza, dolor, fe, sufrimiento, frustración y amor cuya esencia se pierde en otros enfoques investigativos. Aprendemos sobre "la vida interior de la persona, sus luchas morales, sus éxitos y fracasos en el esfuerzo por asegurar su destino en un mundo demasiado frecuentemente en discordia con sus esperanzas de ideales".

La investigación cualitativa es un arte. Los métodos cualitativos no han sido tan refinados y estandarizados como otros enfoques investigativos (pp. 21-23)

Se rescata la idea de que la investigación cualitativa revaloriza al ser humano y no se deja guiar por metodologías estandarizadas, en algunos casos, incluso, compartiendo sus sufrimientos como lo plantean algunas corrientes participativas latinoamericanas, como la investigación acción de la escuela de Fals Borda y, adicionalmente, los investigadores tienen que recurrir al arte de las técnicas y las reflexiones elaboradas in situ como forma adecuada para hacer ciencia, alejando el fantasma instrumentalista de la investigación positivista que sólo aprovecha al ser humano con fines utilitaristas.

Defendiendo un concepto de objetividad la ciencia cualitativa quiere hacer tienda aparte. Sin embargo, se debe observar con atención lo que ocurre en la ciencia como un todo. Los primatólogos, por ejemplo, que cada vez guardan más relación con los antropólogos, se enfrentan a verdaderas protoculturas en las cuales circulan significados, simbolismos, afectividades y una teleología compleja y, sin embargo, no plantean su ruptura con la escuela predominante del positivismo, y ocurre lo mismo con el resto de la etología, sus científicos se ven cada vez más confrontados a sumergirse en la trama de sentidos y vivencias entre animales, pero sin plantear rupturas epistemológicas.

Lo que sucede es que la noción tradicional de objetividad puede ser perfectamente adaptada a la ciencia interpretativa, tal 
y como lo hace la fenomenología, suponiendo una subjetividad personal (la del investigador) que puede captar la esencia del Otro (el sujeto investigado), dejando de lado buena parte de los prejuicios. La fenomenología ha planteado esto, pero no la representación del objeto tal cual es porque ello sería imposible.

El ser humano siempre requiere algún tipo de abstracción o interpretación del mundo que lo rodea porque de otra forma no habría conocimiento, y está claro que en su mente la realidad es una refiguración, una interpretación de la misma. Sin embargo, las traducciones "realistas" son posibles, ya que si nos negamos a esto seríamos incapaces de hacer ciencia y quedaríamos paralizados del todo. Eso quiere decir que podemos conocer sabiendo las limitaciones o la naturaleza de la traducción que se ha hecho. El investigador, puede saber, por ejemplo, la parte del conocimiento que pertenece a las tradiciones, a la Autoridad o al mito, y desde ahí comenzar la crítica de sí mismo, más aún, la ciencia social ha avanzado mucho en esta materia y ahora realiza críticas refinadas sobre posibles creencias arbitrarias metidas en el discurso científico que son ideologías, con lo cual han colaborado el materialismo histórico, el psicoanálisis, la historia de las ideas, la historia de las religiones y la antropología cultural, entre otras. Contando con el conocimiento sobre historia de las religiones, un físico puede diagnosticar acertadamente que la idea de "fuerza" de Newton era pre-científica, que no podía ser justificada a partir de la teoría que desarrolló, y a partir de las de las ideas de Freud sobre la relación entre el Superego y el Dios cristiano, un científico social, puede llegar a evitar en su mente el papel de Dios como consuelo y reforzador de ciertos principios completamente acientíficos; así evitar proyectar dicho consuelo hacia su objeto de estudio (otras personas), y se puede hacer lo mismo con la ideología liberal, vistas las críticas de Marx sobre el tema. Los ejemplos pueden ser multiplicados por cien para demostrar que la ciencia social de hoy ha llegado a una fase de sofisticación en su labor de evitar los prejuicios y prenociones, como las llamaba Durkheim. Ya volveremos sobre este asunto.

Otro aspecto del problema tiene que ver con la construcción de la realidad por parte del sujeto. No se puede afirmar que los colores 
existan, o que los sentimientos formen parte del mundo exterior ajeno a los seres vivos, pero sí que la tierra da vueltas alrededor del sol cada año y que cuando llueve lo que cae del cielo es agua, o que en Sudáfrica hubo racismo en la época del apartheid. Y en este caso, la situación es la misma, se trata de conocimiento y, por lo tanto, de interpretación. El filósofo y pensador español Jesús Mosterín lo explica de forma sencilla: Los colores, por ejemplo, son traductores elaborados a partir de longitudes de onda de la luz que llegan a nuestros ojos, de acuerdo a la luz que resulta visible para nosotros, y si no vemos el ultravioleta es porque esas longitudes fueron ignoradas en nuestro proceso evolutivo (un filtro que seleccionó la especie), de modo que para empezar, el cerebro como entidad biológica realiza una interpretación. Esto lo sabemos, pero no lo podemos percibir, los colores son como alucinaciones que tenemos frente a las longitudes de onda, cualidades que les colocamos a los objetos, pero que no pertenecen a ellos sino que están en nuestros ojos, o mejor, que proyecta nuestro cerebro hacia el mundo exterior. Pero ello no impide -y esto es lo relevante- que seamos capaces de ser realistas frente a dichas longitudes de onda y en relación a los objetos (Mosterín, 1983, pp. 10-11). Es decir, se trata de una ilusión realista, no nos tropezamos con los objetos y sabemos con precisión en qué lugar del espacio se encuentra, de otro modo, hace varios milenios, los animales salvajes de la sabana africana, donde nos tocó evolucionar, nos hubiesen comido y no hubiésemos sobrevivido. De ahí que deben ser llamadas, no tanto alucinaciones, sino "señales realistas" que produce el cerebro y que sirven como "indicadores" frente al mundo.

Con las emociones sucede algo parecido. Si siento miedo frente a un león que se acerca y me encuentro completamente solo en la sabana, no se trata del que el miedo se encuentre en el león como un atributo que le pertenece, como, por ejemplo, el pelaje de su piel, pero sirve para actuar. Si no lo sintiera es probable que me costaría realizar la evaluación que debe hacerse en términos de supervivencia, pasaría a ser indiferente y entonces, correría riesgo mi vida. Al hacerlo, me veo obligado a realizar la evaluación. No es un indicador externo como el color, pero sólo si lo vemos desde un punto de vista, 
al igual que las longitudes de onda, el estímulo sirve para adaptarse. Se podría decir que ambos vienen del exterior aunque de maneras distintas, y la única diferencia es que el miedo es más fácil de elaborar desde la mente.

Desde que abrimos los ojos al mundo estamos interpretando, $\mathrm{y}$ eso no quiere decir que seamos los creadores del mundo exterior. Por ejemplo, hay quienes piensan que con la realidad social es distinto, porque en relación a ella existe lo que se denomina "objetivación". Un grupo de personas piensa algo y como todos lo piensa, entonces, se convierte en realidad, la misma que deriva de que los demás, como mundo externo, son reales frente a cada uno. Si todos creen que las posiciones más altas de la jerarquía social se acompañan del pelo largo en los hombres, esa será la realidad que se observe, y si se cree que el burka tradicional afgano deben utilizarlo todas las mujeres cuando salen a la calle, si todos los creen y piensan que es obligatorio, esa será la realidad que se observe. La objetivación implica que las creencias de los hombres corresponden con la realidad que se vive y que una deriva de la otra, esto es, que tal realidad se transforma para que corresponda con la subjetividad predominante. Fueron ideas que las discutió Marx cuando afirmaba que lo que los hombres eran correspondía con sus obras materiales (según lo que ellos producen, así son), y también varios teóricos asociados a la investigación cualitativa, sobre todo Peter Berger y Thomas Luckman, que en 1966 desarrollaron una teoría convincente sobre el tema. La influencia de esta obra a lo largo del siglo XX y comienzos del XXI, fue enorme.

Esto plantea una paradoja esencial a la discusión sobre la objetividad: si la realidad social corresponde con la subjetividad humana ¿cómo es posible la objetividad? La realidad sería, desde este punto de vista, como un alter ego existente fuera y con vida propia, un correlato objetivo de la subjetividad, y siendo de esa manera ¿cómo puede la mente estudiar el exterior si lo que hay allí son como espejos que la reflejan, un juego de espejos de múltiples caras? El reto, como en un salón de los espejos, es saber dónde realmente se encuentra uno. Aunque Berger y Luckman fueron precisos en los planteamientos que realizaron, las interpretaciones que se han hecho de su obra han sido ambivalentes, y probablemente hundidas en la 
falacia de la homonimia o "falacia de equivocación". Esto quiere decir utilizar los mismos términos, pero con diferentes significados en un mismo discurso. Una de los marcos interpretativos para hacerlo ha sido el liberalismo que lleva a pensar que la realidad social, a) se puede someter a la voluntad individual y b) corresponde con la proyección inmediata de la subjetividad personal y no con el tiempo histórico. Tales enunciados se relacionan más con los libros de autoayuda que con la realidad.

Para estos autores, la realidad social tiene un carácter coercitivo para los individuos, y es construida de forma histórica y colectiva, por lo cual escapa completamente al control individual. Esa realidad no corresponde con los deseos de los individuos, sino que ellos la consiguen ya dada cuando nacen porque viene del pasado. La etnometodología habla, en este caso, de un diálogo o una negociación entre los individuos y la estructura social como forma real de ser en la sociedad, pero aún en estos casos el cambio no es posible si no se cuenta con la acción colectiva. Si se tiene en cuenta el carácter coercitivo, histórico y colectivo de la realidad social se verá que nada tienen que ver con la proyección de contenidos individuales.

Siendo de esta manera, pues, la noción de objetividad queda a salvo tal y como viene siendo discutida. A pesar de que la realidad social pueda ser un resultado histórico de la actividad humana, de subjetividades que van siendo puestas en instituciones o de una praxis social que van determinando la conciencia, se la puede estudiar "objetivamente", es decir, contando con las exigencias epistemológicas de la ciencia. Es más sencillo, como saben los antropólogos, cuando esto es realizado sobre una cultura lejana, pero también es posible sobre una cultura "cercana" como lo demuestran innumerables estudios realizados por antropólogos que se adentran en las urbes modernas, entre minorías o en zonas rurales. Los sociólogos y antropólogos se han infiltrado en sectas religiosas haciéndose pasar por miembros, han explorado normas sociales en los entornos a los cuales pertenecen como lo han hecho los etnometodólogos, han hecho fenomenología sobre sentimientos de duelo, se han aventurado hacia los sistemas simbólicos religiosos en los cuales ellos mismos participan y mucho más. El realismo científico 
en estos casos es posible porque el ser humano puede sobreponerse a las prenociones que pueblan el sentido común, y suspender algunos contenidos del conocimiento tradicional y mítico, pero sobre aplicar de manera equilibrada y cuidadosa las exigencias de algo que renació en el siglo XV y que luego se denominó ciencia. Esto último se refiere a un conjunto de presupuestos o principios sobre cómo llegar a alguna seguridad en cuanto a lo que se cree sobre algo. Claramente, si la objetividad no fuese posible no contaríamos con obras como "Los Argonautas del Pacífico Occidental" (Malinowski, 1986), "Los Hijos de Sánchez" (Lewis, 2012), "Las Estructuras Elementales del Parentesco" y "Mitológicas" (Lévi-Strauss, 1968, 1981) o la misma obra "La Construcción Social de la Realidad" (Berger \& Luckmann, 1968).

En resumen, la construcción social de la realidad no implica que la ciencia social tenga que ser subjetiva. La objetividad no tiene que ver con la metáfora del Espejo o con la tabula rasa de Locke, sino con algo más avanzado. Las interpretaciones no son exactas, pero tanto cometen errores los físicos como los científicos sociales, aunque en este último caso, eso hay que decirlo, los problemas son mayores, ya que las pasiones se desbordan en los temas que son tratados. Si el átomo, y las concepciones del átomo, fuesen ideas de interés para los líderes políticos, es seguro que los físicos tuviesen viviendo los mismos problemas que los teóricos del Estado y el poder.

Esta noción de objetividad fue defendida por el mismo fundador de la investigación acción, Kurt Lewin, quien en una oportunidad comentó a propósito de los criterios de investigación científica que llegó aplicar a su estudio sobre la reducción de conflictos de las minorías en Norteamérica, lo siguiente:

A mí me impresionó vivamente el tremendo efecto pedagógico que, sobre el proceso de adiestramiento, tenían estas reuniones de evaluación que habían sido diseñadas con el propósito de registro científico. La atmósfera de objetividad, la disposición por parte de los profesores a discutir abiertamente sus errores... parecía llevar a un aumento de la comprensión y a implantar ese espíritu de objetividad relajada que en ningún sitio es 
más difícil de encontrar que en el campo de las relaciones intergrupales... (Lewin, 1946, p. 236) (Cursivas nuestras)

La "atmósfera de objetividad" se refiere a la veracidad que puede ser conseguida en reuniones entre los científicos y los ciudadanos comunes que participen de proyectos conjuntos, a partir de los registros científicos y el apego a los datos, algo que es posible conseguir en la Investigación-Acción (IA) si los involucrados se lo proponen. Indudablemente, desde el inicio la Investigación-Acción fue participativa, como lo demuestra la propuesta inicial de Lewin en su propuesta original de 1946, y no refiere sólo a una idea posterior de la época de Orlando Fals Borda. Lo que se ocurrió, en cambio, fue la reinterpretación de la Investigación-Acción según los cánones de una cierta noción de participación emergida en los sesenta en América latina, desde la matriz marxista, y que la entendía a partir de otro tipo de ciencia y objetividad, esto es, la referida a la noción de praxis de la Teoría de la Dependencia y las ideas de Paulo Freire.

\section{¿De dónde viene el problema?}

Parece que las ciencias sociales atravesaron por una etapa, sobre todo en Norteamérica, en la cual casi todo lo que se hacía era cuantitativo y sus defensores la pretendían como modelo. Fue una situación en la que los científicos sociales privilegiaron las matemáticas y los instrumentos estandarizados de recolección de datos como las estrategias, es decir, algo como un paradigma khuniano, un "paradigma epistemológico" para hacer "ciencia normal" a partir de él. Esto ocurrió en la primera mitad del siglo XX (los años 30, 40 y 50) con un grupo de filósofos a la cabeza proveyendo las justificaciones (el Círculo de Viena) o los principios de fondo o las razones por las cuales tenía que ser el modelo. Se argumentaron tesis relativas al mal uso del lenguaje y a lo que la filosofía y el pensamiento tradicional habían propuesto, pero que se encontraba vacío de significado. En efecto, se dijo, muchas de las tesis del pensamiento universal no tenían sentido.

El momento fue animado por una serie de innovaciones en la estadística que se ensayaron desde la bioestadística hasta las ciencias sociales y entre las que cabe recordar el Coeficiente de Correlación de 
Pearson (1898), la $t$ de Student (1908), los grados de libertad (1922), el Chi-Cuadrado (1900), la teoría del muestreo (1934) y la Escala de Likert, entre otras. Un autor comenta que, por entonces, nacieron los conceptos de parámetro, estadístico, varianza, hipótesis nula, test de significancia, nivel de significancia, aleatorización, etc. (Yañez, 2000, p. 9). Todo esto tiene relación con lo que anteriormente ha sido entendida como la matemática relativa a la relación entre variables.

Sobre la matemática relaciona con los algoritmos, hubo casos como el del gran pensador francés Claude Levi-Strauss, que mostró entusiasmo por el tema y escribió sobre las posibilidades de las matemáticas para, en este caso, contribuir con el desarrollo pleno del estructuralismo, la corriente que él mismo había fundado. En un artículo de 1954 contaba la forma cómo se podía proceder para las "matemáticas cualitativas" (Levi-Strauss, 2008: 21). Las reglas del matrimonio y del incesto no podían ser tratadas como cantidades, pero si como reglas lógicas entre clases finitas; la idea se le había ocurrido al saber de la propuesta de un joven de este campo que planteó el problema en esos términos:

Lo único que necesitaba era lo siguiente: en primer lugar, que los matrimonios observados en una sociedad determinada pudieran reducirse a un número finito de clases; y en segundo lugar, que esas clases estuvieran unidas entre sí por relaciones determinadas, por ejemplo que existiese siempre la misma relación entre la "clase" de matrimonio del hermano y la "clase" de matrimonio de la hermana, o entre la "clase" de matrimonio de los padres y la "clase" de matrimonio de los hijos. (Levi-Strauss, 2008, p. 22).

Y continuaba: "A partir del momento en que se disponía de esos elementos, todas las reglas del matrimonio en una sociedad determinada se podían formular en ecuaciones susceptibles de ser tratadas con métodos de razonamiento rigurosos y verificados...". Insistía en que las normas de relación social no eran cantidades y no podían ser abordadas por las matemáticas tradicionales, como el álgebra, pero si por unas más avanzadas fundadas en la lógica formal, al menos, para las reglas elementales del parentesco. 
De todos modos, luego de un tiempo mucha gente comenzó a sentir insatisfacción, gran insatisfacción, sobre todo en la medida en que las corrientes subterráneas del racionalismo y el idealismo comenzaban a creer y a ganar adeptos, y teorías como la de Max Weber se popularizaban. Intelectuales como Wright Mills hicieron reclamos eficaces en obras como "La Imaginación Sociológica", porque los resultados del período de matematización habían sido mediocres y no se habían realizado hallazgos importantes para las ciencias sociales, sino por el contrario, parecían detenidos. Las revisiones que se hicieron, por ejemplo, en el campo de la investigación evaluativa en educación (investigación para medir resultados) revelaban que los diseños estadísticos no eran capaces de evitar la subjetividad a pesar de lo detallado del análisis matemático, o lo que es lo mismo, que dicho análisis por muy sofisticado que fuera, sólo servía para ocultar resultados mediocres o limitaciones que pertenecían a los investigadores. Así lo expresa Aliaga en un buen artículo:

Los estudios (revisados) adolecen de una excesiva atención al uso de elaborados métodos estadísticos para intentar solventar las deficiencias en los diseños de investigación. Los métodos de análisis estadístico no pueden corregir los fallos en la conceptualización, diseño y ejecución de los estudios (Meyer \& Conrad, 1992, citado por Aliaga, 2000, p. 302)

De la etapa de "matematización" no quedaron las "leyes" o tendencias deterministas que se esperaban, y sí quizás el sentimiento de que no era un camino correcto. Se aspiraba que la estadística descubriera tales "leyes", pero eso no sucedió porque esta rama, como se ha dicho anteriormente, no tiene que ver con las relaciones sociales.

La investigación cualitativa pareció, entonces, una reacción tardía a un tipo de ciencia, pero con el problema que se desarrolló como un alter ego o una imagen en contrario. Si el positivismo lógico hablaba de "experiencia" las nuevas corrientes lo hacían en términos de la "vivencia" (la propuesta de Dilthey como la piedra angular sobre la cual levantar la nueva ciencia), si se hablaba del "sentido" como algo ausente de la realidad, la investigación cualitativa lo ponía como 
omnipresente, si se argüía que la realidad podía ser estudiada sin recurrir a los significados se proclamaba la sociología comprensiva de Weber, si los positivistas presuponían una realidad externa ya constituida se insistía en la realidad interna construida por el sujeto, y si se hablaba de matemática como la forma de lenguaje más adecuada se le oponía el "discurso desde el punto de vista del sujeto" como lo correcto. Lo que se hizo, pues, fue una inversión de valores. Dilthey, por ejemplo, había reclamado para las ciencias del espíritu la vivencia del Otro y no simplemente tener experiencia directa con él al estilo de la ciencia tradicional (es decir, una ruptura con el empirismo):

En una palabra, mediante la vivencia el hombre tiene conocimiento del mundo humano. De aquí concluye Dilthey que la estructura teórica de las ciencias humanas difiere de las de las ciencias de la naturaleza: esta es explicativa y aquella comprensiva (Ortega, 1983, p. 217) (cursivas nuestras)

Sin embargo, no se podía responder al objetivismo cosificador del positivismo con la afirmación de que todo en el mundo humano era simbólico y vivencial, o que el subjetivismo (la subjetividad humana) era la raíz de todo el orden social (para relegar lo material a un segundo plano), o como muchos proclamaron equivocadamente a partir de Clifford Geertz, que la verdadera opción tenía que ver con la "ciencia interpretativa":

Creyendo con Max Weber que el hombre es un animal inserto en tramas de significación que él mismo ha tejido, considero que la cultura es esa urdimbre y que el análisis de la cultura ha de ser por lo tanto, no una ciencia experimental en busca de leyes, sino una ciencia interpretativa en busca de significaciones (Geertz, 2003, p. 20)

Desde luego que a proclamas como esta, Marvin Harris, un materialista convencido, opuso la distinción entre la antropología social y cultural, o entre "las reglas mentales para actuar y hablar compartidas por los miembros de una determinada sociedad" y "las actividades culturalmente determinadas que realizan con sus cuerpos". 
Algunos antropólogos, empero, restringen el significado de «cultura» exclusivamente a las reglas mentales para actuar y hablar compartidas por los miembros de una determinada sociedad. Estiman que estas reglas constituyen una especie de gramática de la conducta y consideran las accionescomo fenómenos deíndole «social» más que «cultural». Ésta es la distinción que algunos antropólogos tratan de establecer cuando diferencian la antropología social de la cultural (Goodenough, 1970). Ninguna confusión puede derivarse de la definición más inclusiva, siempre que se tenga el cuidado de indicar si se está hablando de las ideas y sentimientos culturalmente determinados pertenecientes a la vida mental de la gente, de las actividades culturalmente determinadas que realizan con sus cuerpos, o de ambas cosas (Harris, 2004, p. 40)

Dado que ciertamente, no es lo mismo mover el cuerpo que decodificar mensajes en la mente, y dado que el sustrato material sobre el que levanta la vida social es innegable, Harris se da cuenta de que la definición de Edward Tylor (la más conocida en antropología) va mucho más allá que las definiciones subjetivistas. Por lo cual, no se puede tratar sólo de significados. Si en el análisis de dos pueblos tribales de la polinesia, uno con mayor desarrollo en tecnología militar, complejidad social y técnicas agrícolas que el otro, desarrolla la condición de cazadores-recolectores, al no producir "excedentes de cultivos disponibles para su redistribución o almacenamiento", lo cual impide, como se sabe, el mantenimiento y alimentación de "especialistas artesanos no cazadores, ejércitos, burócratas y jefes", eso constituye una reflexión perfectamente válida y pertinente.

Al no haber otras islas accesibles para colonizar, los marioris tuvieron que permanecer en las Chatham y aprender a soportarse los unos a los otros. Para ello renunciaron a la guerra y redujeron los posibles conflictos derivados de la superpoblación, castrando a algunos varones de corta edad. El resultado fue una población pequeña y no belicosa dotada de tecnología 
y armas sencillas y sin liderazgo ni organización fuertes"

(Diamond, 2006, p. 66)

En este caso el resultado, una "población pequeña y no belicosa dotada de tecnología y armas sencillas y sin liderazgo ni organización fuertes", un factor derivado de la "falta de espacio" y la "carencia de agricultura". El ser humano también es un cuerpo material que interactúa con un mundo material y a lado de la lógica simbólica se encuentra la lógica mandada por la "cultura material".

Es probable, entonces, que los defensores de la ciencia social como exclusivamente interpretativa, holística y naturalista hayan exagerado un poco, lo cual puede verse con más claridad cuando se la pretende como una cosmovisión sustitutiva de la anterior; es decir, como el desarrollo de un paradigma inverso, olvidar los datos cuantitativos sería una meta, así como los procedimientos que llevan a su recolección y análisis. Ahora bien ¿de qué modo conseguir una superación y no la inversión?

\section{El prejuicio del "ordinarismo"}

Si la ciencia depende de la "Mirada" (en sentido antropológico) o de una lectura de la realidad, lo que queda en pie es recurrir a criterios de "seriedad", rigor y rigurosidad en dicha lectura. Sin embargo, el punto final de este hilo de reflexión termina casi siempre en el "ordinarismo", como lo ha llamado Mario Bunge, o sea, la creencia según la cual la ciencia es un conocimiento "ordinario" como cualquier otro, que no tiene nada de especial, sino que sólo es diferente y no posee criterios de demarcación que permitan distinguirlo del resto de formas de conocimiento (Bunge, 1998, pp. 111-120).

Ahora bien, es cierto que el saber científico es "ordinario" en un sentido, pero no en todos, es decir, se trata de una idea ambigua aprovechada para decir muchas cosas al mismo tiempo y promover malos entendidos, como algo perteneciente al mundo de las representaciones y las ideologías mas no de la epistemología o de la "antropología de la ciencia". Es cierto que un indígena yanomami puede saber más sobre su entorno natural que cualquier científico 
occidental, y conocer las plantas medicinales más que cualquiera, con secretos que todavía la ciencia occidental no conoce, pero ello sólo probaría, una vez, que el método científico no es un criterio de demarcación. Y las taxonomías empleadas por la ciencia para dar cuenta de los animales y las plantas pudieran ser peor que las empleadas por alguna etnia africana como lo afirmó Feyerabend (1986) en su famosa obra ("Tratado contra el Método") o , que la ciencia sigue estrategias de abstracción semejantes a las empleados por cualquier nativo africano.

Para realizar un acercamiento al problema se debe recurrir a las ideas iniciales que provocaron la revolución de la ciencia luego del Renacimiento y citar a los antiguos. Baruch de Spinoza, por ejemplo, que acompañó a Descartes en las ideas más importantes del siglo XVII, lo entendía bien en 1661, y con una claridad meridiana.

Es evidente que, aparte de ser una fuente insegura, de oídas no conocemos la esencia de ninguna cosa, como se ve en nuestro ejemplo. Ahora bien, como la existencia singular de una cosa no es conocida si no se conoce su esencia (según veremos más tarde), toda certeza adquirida de oídas debe ser excluida de las ciencias. Pues mientras no se dé un acto previo del entendimiento, de oídas simplemente nadie puede ser convencido" (Spinoza, 1968, p. 77) (Cursivas nuestras)

Spinoza ponía sobre la mesa la idea clave, a saber, la certeza o seguridad debe excluir el conocimiento de "oídas". Como se sabe, esto ya había sido dicho en lo esencial por Locke, Hume y Bacon en el sentido de que las certezas adquiridas de oídas (de rumores, de lo que dice la gente en la calle y de aquello que enseñan las tradiciones, también la Autoridad) debían ser excluidas. Suponiendo que la ciencia pertenece a un gran movimiento cultural, donde ella es uno de los resultados, que no se trata de un movimiento lógico o filosófico, es posible afirmar que de lo que se trata es de lo siguiente: hubo una vez un día en el que los intelectuales dejaron de creer en estas formas de conocimiento antiguas, en esta manera de demostrar las cosas, y quisieron experimentar por cuenta propia y demostrar en base al comportamiento observable, sumergiéndose en la realidad 
ellos mismos. Dejaron de leer libros y se dedicaron a constatar en la realidad las ideas, un momento en el que se sintió la necesidad de convertir en una premisa insustituible el canon cartesiano de "dudar de todo", y refundar el conocimiento.

El carácter de revolución cultural es algo que hemos olvidado de la mano del mundo moderno y ha sido un error. El indígena Yanomami y el científico pueden llegar a la misma verdad y lo que sucede con la ciencia es que se niega a considerar el conocimiento tradicional como válido, y decide siempre partir de cero para conseguir sus propias verdades, esto es, sometiendo las ideas a sus propias exigencias y demostraciones. Es esto lo pueden entenderse como la "seguridad científica" (cómo hace un investigador para estar seguro) y es la meta general de la ciencia. Si al final ocurre que la creencia que tiene el yanomami y la de él es la misma, eso no es lo importante, el estatuto del saber científico se toma el trabajo de someter sus ideas a exigencias que ha desarrollado a lo largo de los siglos y en todo el proceso, la comunidad científica es el árbitro. En su interior no caben los conocimientos míticos, tradicionales o del sentido común, validados por el sentido común. La ciencia posee sus criterios de validación o "criterios de verdad".

El conocimiento adquirido a través de las tradiciones (una parte del cual Bacon llamaba los "Ídolos de la Tribu"), la recurrencia a la "Autoridad" para validar el conocimiento (a los clásicos, sus libros y a los grandes pensadores del pasado), y el consenso social, fueron abandonados en los días del Renacimiento. En lugar de buscar en los libros, mejor escrutar directamente con un telescopio (como lo hizo Galileo), en lugar de buscar en esos mismos libros la naturaleza de la luz, realizar experimentos con ella, y en vez de aceptar la sociedad como un algo natural, la "ingeniería social" a través de formas de gobierno resultado de la planificación de los hombres, como lo pensó la Revolución Francesa y la Revolución Rusa de 1917. La posibilidad de una ingeniería social, por cierto, se derivaba de las premisas anteriores, a saber, si se iba a olvidar el conocimiento tradicional también las formas tradicionales de sociedad, la "tecnología social" era la consecuencia directa del saber práctico (para la noción de "tecnología social" ver Vignolo, 2002; Pereira \& Chirinos, 2004). 
Se comprende mejor todo esto al pensar en la anécdota atribuida a Francis Bacon sobre la diferencia entre ciencia y formas tradicionales de conocimiento. Él cuenta lo ocurrido en un monasterio durante una disputa entre varios monjes, donde uno de ellos representaba el pensamiento científico, se sentaron a discutir sobre las muelas del caballo:

En 1432 surgió una gran polémica entre los hermanos acerca del número de muelas que tiene el hocico del caballo. Trece días duró el calor de la disputa. Consultáronse todos los libros y crónicas científicas, y púsose de manifiesto una poderosa y maravillosa erudición como nunca se había escuchado en aquella región. En el día decimocuarto un joven fraile de grata prestancia solicitó permiso a sus superiores para decir algo. Y ante la sorpresa de los disputantes, cuya profunda sabiduría lo había irritado, les pidió inclinarse y adoptar una postura vulgar y extraña para ellos; les dijo que mirasen dentro del hocico abierto de un caballo para encontrar la respuesta que buscaban. Tales palabras hirieron profundamente la dignidad de los frailes. Se irritaron en extremo y vociferando, echáronse sobre él, lo azotaron en las posaderas y lo arrojaron fuera. Lo hicieron porque estaban seguros de que Satanás lo había tentado para que declarara formas sacrílegas y desconocidas de encontrar la verdad, contrarias a las enseñanzas de los padres. Al cabo de muchos días de discordias, la paloma de la paz volvió a la asamblea, y todos los frailes estuvieron de acuerdo en que el problema era un misterio insoluble, pues no contaban con pruebas históricas y teológicas al respecto. Ordenaron pues, que se asentase esta declaración (Kenneth, 1934, citado por Ary, Jacobs, \& Razavieh, 1989, p. 5)

Para la ciencia lo más normal sería una constatación directa en la realidad de las preguntas que se realizan, pero no para el pensamiento tradicional teológico y medieval centrado en la exégesis y la Autoridad. Para éste, la verdad debía ser buscada en los libros, 
entre los grandes sabios, y -como se afirma en la anécdota-, si no era conseguido ahí, entonces no era digno de ser tenido como conocimiento. Se debe notar el alejamiento de esta postura, incluso, respecto del humanismo que logró nacer de la escolástica en la que el hombre sí era el centro del conocimiento, es decir, para las posturas más tradicionales la Autoridad (y Dios) eran el centro, por lo cual no podía el ser humano conseguirlo de forma directa, y esa era la razón por la cual se escandalizaron los monjes con la propuesta de ir a verificar directamente en el hocico de los caballos.

Ahora bien, la aclaratoria que esto produce respecto al ordinarismo es que la ciencia sí posee "criterios de validación" o "criterios de verdad" distintos, que emergieron con los primeros empiristas o que ellos intentaron concientizar. Alvin Toffler los llama los "filtros de verdad".

... la batalla por la verdad no está confinada a la ciencia.

Distintos grupos sociales están tratando activamente, por unas u otras razones, de gestionar nuestras mentes desplazando los filtros de la verdad a través de los que, a nuestra vez, vemos el mundo, las pruebas que usamos para separar lo verdadero de lo falso (Toffler, 2007, p. 203).

Dichas pruebas son diferentes para cada cultura, cada grupo social, cada religión, y todos se encuentran en competencia. Lo que hizo la ciencia a partir del Renacimiento fue construir unos criterios que la separaron históricamente del resto, con unos criterios que desde esos días pone en práctica. Dentro de esos criterios se encuentran: a) el dejar de creer de oídas, una actitud escéptica o de incredulidad ante el mundo, b) un realismo a toda prueba que conduce a sus practicantes a la inmersión total en sus lugares de estudio, c) un empirismo de base para las ideas que deben ser demostradas y, d) un auto-reforzamiento progresivo que la lleva a nutrirse sólo de sí misma. No es por tanto un conocimiento más, sino una vanguardia con sus propios criterios de verdad y que ha demostrado su eficacia en la construcción de tecnologías materiales y no materiales. La ciencia ha demostrado ser realista, para no decir objetiva, y tomarse muchas precauciones antes de creer en algo o 
dejarlo entrar en su acervo de conocimiento. No es un conocimiento de la misma naturaleza que el conocimiento religioso, porque tiene criterios de validación completamente distintos, no es conocimiento de sentido común, por la misma razón y, por ello, la ciencia tiene derecho a su propia identidad.

Las acusaciones de Feyerabend quedan atrás, la ciencia no es lo mismo que las teorías africanas indígenas sobre la realidad, porque tales teorías no han sido conseguidas con sus criterios de validación, y en esto radica la diferencia esencial. Los criterios de validación del saber mítico y tradicional son distintos, son otros. La ciencia no se parece al conocimiento tradicional porque de hecho, ya en los días de Bacon se decidió rechazar tal forma de conocimiento, y en los días de Descartes, ya era patente la duda respecto a todo lo que hasta ese momento se sabía.

\section{Hacia una ciencia social avanzada... (A modo de conclusión)}

La ciencia es una aplicación combinada de estos criterios (ya esbozados) y no de algún método en particular, o caracterizada por factores como la Falsabilidad. En un sentido estricto, todas las formas de conocimiento y culturas tienen su principio de Falsabilidad y no sirve de nada insistir en que sólo la ciencia hace eso. Pero, por otro lado, conocer es interpretar porque no cabe otra posibilidad. En el nivel más básico el cerebro interpreta de acuerdo a su biología y es la razón por la cual percibimos los colores, aunque no existen en la realidad, y en los niveles más elevados, interpretamos la realidad de acuerdo a la cultura a la cual pertenecemos.

Pero a pesar de todo, la ciencia objetiva es posible. Objetividad significa hacer una interpretación "realista" al modo como la ciencia lo exige, y hacerlo cumpliendo con criterios. La eficacia del realismo, que es como debe ser llamada la objetividad, parte de la duda cartesiana frente al conocimiento mítico anterior, acumulado por la sociedad, y por los otros criterios validación del conocimiento, de tal manera que la ciencia moderna es un subproducto cultural de esto. 
La noción típica de objetividad como "hablar desde el punto de vista del objeto" puede ser traslada de esta manera, no como algo absoluto, sino como un esfuerzo que hay que hacer si se está dentro de la ciencia; afirmar que es imposible no conduce a nada, ni tampoco es realista. Es cierto que la realidad humana incluye lo vivencial y lo simbólico, pero esto no separa a las ciencias interpretativas del resto de la ciencia. La objetividad que se encuentra del lado dentro de la ciencia no es positivista ni interpretativa, la prédica del Ordinarismo no dice nada.

Si se afirma que la investigación cualitativa es subjetiva, se deben hacer varias aclaratorias. No se trata de que no puede ser "realista", sino que ella realiza una lectura de los Otros teniendo en cuenta sus subjetividades, con sus interpretaciones, voliciones y simbolismos incluidos. Si se quiere afirmar que el sujeto construye la realidad, de todas maneras es cierto que la tierra da vueltas alrededor del sol, y no al revés, que la electricidad está causada por el movimiento de los electrones y que entre los Trobriand existe el Kula como una forma de intercambio social compleja, o que hay sociedades matrilineales y patrilineales, o que el proceso de colonización europea de África ocurrió de tal modo que el racismo fue uno de sus resultados. Por lo que, si el sujeto construye su realidad, no será en el sentido de que sin su interpretación estas cosas dejarían de suceder.

Una ciencia cualitativa avanzada debe identificarse con una ciencia social avanzada y realizar sus aportes dejando atrás las limitaciones de la confrontación con el positivismo. Tales limitaciones se relacionan con el Ordinarismo, el subjetivismo en el sentido de que la realidad humana sólo está conformada por elementos subjetivos (p. ej. los símbolos) y el presupuesto de que el carácter único de la realidad humana debe llevarnos a una ruptura epistemológica con el resto de la ciencia.

La ciencia social avanzada se encuentra en el punto donde percibimos, por un lado, que la realidad humana tiene un aspecto referido a una estructura simbólica en la cual se inserta el comportamiento humano y la subjetividad, pero, por el otro, que otros aspectos tienen que ver con el sustrato material subyacente que plantea lógicas a las sociedades y que, adicionalmente, sobre 
ello se pueden crear algoritmos; que existen diferentes "lógicas" que atraviesan la sociedad y la constituyen como un resultado; que, como siempre se ha dicho, el ser humano es un ser biopsicosocial (no solamente subjetivo) y que, por lo tanto, al tener cuerpo y necesidades materiales, interactúa con el mundo material, razón por la cual deben tener cabida corrientes de pensamiento que estudien esos aspectos. El enfoque comprensivo, por tanto, no puede ser una cosmovisión, y para quienes ha creído en eso, han cometido un grave error.

Esa ciencia se encuentra donde recibe su aclaración final la epistemología de base, o sea, que la ciencia comienza donde se intersectan todos los criterios anteriores y donde las "pruebas" terminan siendo la meta. Los científicos deben sentarse a discutir pruebas, es decir, a debatir con los registros científicos en las manos y probar a realizar inferencias sobre ellos, y no simplemente a especular y menos sobre ideologías sociales promovidas desde el poder. Hacer ciencia no es como hacer poesía o escribir novelas como algunos han planteado para la etnografía, ni siquiera en el sentido de una estrategia expositiva para el lector, la interpretación del etnógrafo no es literatura, sino conocimiento que cumple con el status de la ciencia. La integración metodológica significa que el paso hacia adelante debe ser dado en esa dirección.

\section{Referencias}

Aliaga, F. (2000). Validez de la Investigación Causal: Tipologías y Evolución. Bordon, 52, 301-321.

Ary, D., Jacobs, L. C., \& Razavieh, A. (1989). Introducción a la investigación pedagógica. México; San Juan: McGraw-Hill.

Berger, P., \& Luckmann, T. (1968). La construcción social de la realidad. (S. Zuleta, Trad.). Buenos Aires: Amorrortu.

Bunge, M. (1998). Sociología de la ciencia. Buenos Aires: Editorial Sudamericana.

Cipolla, C. M. (1981). Historia económica de la Europa preindustrial. Madrid: Alianza Editorial.

Cook, T. D., \& Reichardt, C. S. (1986). Métodos cualitativos y cuantitativos en investigación evaluativa. Madrid: Morata. 
Diamond, J. (2006). Armas, Gérmenes y Acero (Tercera Edición). Caracas, Venezuela: Randon House Mandadori.

Feyerabend, P. K. (1986). Tratado contra el método: esquema de una teoría anarquista del conocimiento. Madrid: Tecnos.

Geertz, C. (2003). La Interpretación de las Culturas. Barcelona: Gedisa.

Goodenough, W. H. (1970). Description and Comparison in Cultural Anthropology. Chicago: Aldine.

Harris, M. (2004). Antropología Cultural. Madrid: Alianza Editorial. Recuperado de http://faces.unah.edu.hn/arqueo/images/stories/docs/Documentos_en_Linea/Marvin\%20Harris\%20Antropolog\%EDa\%20Cultural.pdf

Herodoto. (2006). Los Nueve Libros de la Historia. (B. Pou, Trad.). EbookLibris (tomado de Wikisoruce.org. Recuperado de http://www.enxarxa.com/biblioteca/HERODOTO\%20Historia\%20_Pou_.pdf

Lévi-Strauss, C. (1968). Mitológicas. México, D.F.: Fondo de Cultura Económica.

Lévi-Strauss, C. (1981). Las estructuras elementales del parentesco. Barcelona: Editorial Paidós.

Lévi-Strauss, C. (2008). Las Matemáticas del Hombre. El Correo de la UNESCO, (5), 21.

Lewin, K. (1946). La investigacion-accion y los problemas de las minorias. Revista de Psicología Social, 3(2), 229-240.

Lewis, O. (2012). Los hijos de Sánchez. Autobiografía de una familia mexicana. México, D.F.: Fondo de Cultura Económica.

Malinowski, B. (1986). Los argonautas del Pacífico occidental. Barcelona: Planeta-Agostini.

Mosterín, J. (1983). Grandes temas de la filosofía actual. Madrid: Aula Abierta Salvat.

Ortega, J. M. (1983). Wilhelm Dilthey: Crítica de la Razón Histórica. En Historia del Pensamiento (pp. 215-218). Barcelona, Esp.: Ediciones Orbis. 
Padrón, J. (2007). Tendencias epistemológicas de la investigación científica en el siglo XXI. Cinta de Moebio. Revista de Epistemología de Ciencias Sociales, (28). Recuperado de http:// www.revistas.uchile.cl/index.php/CDM/article/viewArticle/25930

Pereira, L. (2000). El sentido de los museos (Estudio sobre las representaciones de los museos y lo antropológico en Maracaibo y los Puertos de Altagracia. Edo. Zulia-Venezuela). Boletín Antropológico, 3(50), 29-52.

Pereira, L., \& Chirinos, O. (2004). La Nueva Ingeniería Social: Notas para una Epistemología Avanzada de las Ciencias Sociales Aplicadas. Reforma y Democracia, (28), 207-232.

Rincón, I., Gamero, M., \& Ortín, N. (1986). La Universidad del Zulia en el proceso histórico de la región zuliana (1ra. ed). Maracaibo: Universidad del Zulia, Vice-Rectorado Académico.

Ruiz Bolívar, C. (2008). El enfoque multimétodo en la investigación social y educativa: una mirada desde el paradigma de la complejidad. Teré: revista de filosofía y socio-política de la educación, (8), 13-28.

Spinoza, B. (1968). La Reforma del Entendimiento. Maracaibo, Venezuela: Universidad del Zulia.

Taylor, S. J., Bogdan, R., \& Piatigorsky, J. (1987). Introducción a los métodos cualitativos de investigación: la búsqueda de significados. Barcelona: Paidós. Recuperado de http://mastor.cl/ blog/wp-content/uploads/2011/12/Introduccion-a-metodos-cualitativos-de-investigaci\%C3\%B3n-Taylor-y-Bogdan.-344-pags-pdf.pdf

Toffler, A. (2007). La Revolución de la Riqueza. Caracas, Venezuela: Randon House Mandadori.

Vignolo, C. (2002). Sociotecnología: Construcción de capital social para el Tercer Milenio. Reforma y Democracia, (22). Recuperado de http://old.clad.org/portal/publicaciones-del-clad/ revista-clad-reforma-democracia/articulos/022-febrero-2002/0041013 
Wittgenstein, L. (2007). Tractatus logico-philosophicus. (L. M. Valdés Villanueva, Trad.). Madrid: Tecnos.

Yañez, S. (2000). La Estadística una ciencia del siglo XX. R.A. Fisher, un genio. Revista Colombiana de Estadística, 23(2), 1-14. 